\title{
Образование оксидного поверхностного слоя и его влияние на рост эпитаксиальных нитевидных нанокристаллов кремния
}

\author{
(C) В.А. Небольсин, Н.А. Свайкат , А.Ю. Воробьев, Т.А. Перепечина, Л.В. Ожогина
}

Воронежский государственный технический университет, 394026 Воронеж, Россия

"E-mail: vcmsao13@mail.ru

Поступила в Редакцию 12 апреля 2021 г.

В окончательной редакции 19 апреля 2021 г.

Принята к публикации 19 апреля 2021 г.

\begin{abstract}
Установлено, что при выращивании нитевидных нанокристаллов $\mathrm{Si}$ в потоке $\mathrm{H}_{2}$, не подвергшемся дополнительной очистке от остатков $\mathrm{O}_{2}$ и паров воды, на поверхности кристаллов и на ростовой подложке образуются слои $\mathrm{SiO}_{2}$. Из-за присутствия оксидного поверхностного слоя рост нитевидных нанокристаллов $\mathrm{Si}$ заторможен, кристаллы характеризуются сильной морфологической неустойчивостью. Определены термодинамические условия образования поверхностного оксидного слоя и его влияния на рост нитевидных нанокристаллов Si. При температурах синтеза 750-1400 K нитевидные кристаллы Si термодинамически неустойчивы в газовой фазе, содержащей любые ощутимо малые концентрации $\mathrm{O}_{2}$, и при благоприятных кинетических условиях $\mathrm{Si}$ должен всецело превращаться в оксид. Термическая диссоциация и водородное восстановление $\mathrm{SiO}_{2}$ в условиях роста кристаллов $\mathrm{Si}$ практически неосуществимы.
\end{abstract}

Ключевые слова: нитевидные нанокристаллы, кремний, рост, оксидный слой.

DOI: 10.21883/FTP.2021.09.51297.27

\section{1. Введение}

В настоящее время нитевидные нанокристаллы (ННК) полупроводниковых материалов (нанопроволоки) имеют большой потенциал для практического применения. Это обусловлено целым рядом их преимуществ, среди которых, естественный механизм эффективной релаксации упругих напряжений и возможность формирования гетероструктур ННК из систем материалов с большим рассогласованием по параметру кристаллической решетки $[1,2]$; возможность переключения кристаллической фазы в процессе роста, например, по типу сфалерит $\rightarrow$ вюртцит $[3,4]$; возможность создания квазиодномерных объектов, имеющих характерные поперечные размеры порядка длины волны де Бройля и квантово ограничивающих движение электронов в двух направлениях [5,6]; возможность создания фотоэлектрических структур, у которых глубина поглощения света в полупроводнике и длина диффузии неосновных носителей заряда не являются конкурирующими конструктивными параметрами [7-9]; наличие высокого термоэлектрического эффекта [10]; высокая плотность холодной эмиссии электронов с остриев ННК [11] и др. Однако сдерживающим фактором применения ННК являются проблемы их управляемого выращивания. Так, например, непреднамеренное окисление ростовой $\mathrm{Si}$-подложки, частиц металла-катализатора и боковой поверхности эпитаксиальных НHК Si приводит к неустойчивости кристаллизационного процесса, а в отдельных случаях, вообще, может блокировать рост кристаллов [12]. Понимание механизма образования поверхностного оксидного слоя и его влияния на рост НHК Si может быть важным шагом в осуществле- нии воспроизводимого, низкотемпературного и управляемого роста.

Цель настоящей работы - определить термодинамические условия образования поверхностного оксидного слоя и его влияние на рост НHК Si.

\section{2. Методика эксперимента}

Выращивание ННК Si осуществляли в эпитаксиальной установке проточного типа („Изоприн“, АО „НИИПМ“) в процессе водородного восстановления $\mathrm{SiCl}_{4}$ при температуре от 1300 до $1400 \mathrm{~K}$. Молярное соотношение $\left[\mathrm{SiCl}_{4}\right] /\left[\mathrm{H}_{2}\right]$ задавалось в интервале от 0.05 до 0.10 .

Используемый для выращивания ННК кремния $\mathrm{H}_{2}$ имел точку росы $215 \mathrm{~K}$ и содержал $1 \cdot 10^{-3} \% \mathrm{O}_{2}$, поэтому он подвергался дополнительной очистке и осушке в газоочистительной установке („Цветхром-60“, ООО „Цветхром“). Установка позволяла снизить содержание $\mathrm{O}_{2}$ до $1 \cdot 10^{-5} \%$ и точку росы до $200 \mathrm{~K}$, что исключало возможность окисления $\mathrm{Si}$ при температурах выращивания ННК.

В специальных экспериментах $\mathrm{SiO}_{2}$ на поверхности ННК, а также оксиды металлов-катализаторов и оксидную пленку на ростовой подложке получали термическим окислением в присутствии остатков кислорода в процессе выращивания, для чего в ростовую камеру подавали $\mathrm{H}_{2}$ без дополнительной очистки от $\mathrm{O}_{2}$.

Ростовыми подложками служили полированные монокристаллические пластины $\mathrm{Si}$ с кристаллографической ориентацией $\{111\}$. Непосредственно перед выращиванием ННК поверхность пластин обрабатывалась водородом при температуре $1400 \mathrm{~K}$ в течение 10 мин 
(при этом происходило восстановление $\mathrm{Si}$ из поверхностного слоя оксида, образованного при контакте с атмосферой воздуха), а затем хлористым водородом для стравливания нарушенного слоя $\mathrm{Si}$. Содержание $\mathrm{HCl}$ в $\mathrm{H}_{2}$ составляло $1-2$ об \% , при этом достигалась скорость травления 1-2 мкм/мин. В отдельных экспериментах указанная обработка не проводилась. Рост ННК осуществлялся с участием каталитических частиц, представляющих собой пересыщенный раствор кристаллизуемого вещества в жидкофазных каплях $\mathrm{Au}$ ограниченного объема. Диаметры наночастиц составляли от 50 до 500 нм. Размеры каталитических частиц определялись по изображениям частиц сплава $\mathrm{Au}-\mathrm{Si}$ на поверхности ростовой подложки в сканирующем зондовом микроскопе Nтегра Прима (С3М) [13]. Средний размер частиц составлял 180-200нм. Выбор Аu в качестве катализаторов пар-жидкость-кристалл (ПЖК)роста ННК определяется тем, что оно образует с $\mathrm{Si}$ низкотемпературную эвтектику и стойко к окислению. Нанесение частиц $\mathrm{Au}$ на поверхность Si-пластины осуществлялось напылением тонкой пленки металла с последующим нагревом и разбиением на отдельные капли. Разбиение пленки на отдельные капли проводили путем ее отжига в течение 5-20мин при температуре $1300-1400 \mathrm{~K}$ в высокотемпературной печи Naberterm (Германия) в атмосфере очищенного $\mathrm{H}_{2}$. Поскольку пленка металла термодинамически неустойчива, она под действием поверхностного натяжения хаотично разбивается на отдельные капли.

Морфологические и структурные исследования выращенных кристаллов проводились методами металлографии, сканирующей зондовой („Nтегра Прима“, НТ МДТ) и растровой электронной микроскопии, энергодисперсионного рентгеноспектрального микроанализа (JSM-6380LV, JEOL). Электронографические исследования оксидных пленок проводили на электронографе ЭМР-100 (ЗЭМ, Сумы).

\section{3. Результаты и их обсуждение}

При выращивании ННК Si в присутствии наночастиц $\mathrm{Au}$ в потоке $\mathrm{H}_{2}$, не подвергшемся дополнительной очистке от остатков $\mathrm{O}_{2}$, и в котором присутствуют следовые остатки паров воды, на поверхности кристаллов и ростовой подложке образуются оксидные слои толщиной 0.5-1.5 нм. Согласно результатам энергодисперсионного микроанализа полученных образцов, элементный состав поверхностной оксидной пленки ННК может быть выражен как $\mathrm{SiO}_{x}$ (где $x$ принимает значения от 1 до 2), что соответствует составу контрольных кварцевых образцов (рис. 1, $a, b)$. На рис. 1, с приведена электронограмма участка оксидного поверхностного слоя. На электронограмме наблюдается диффузное гало, указывающее на присутствие аморфной фазы, поскольку $\mathrm{SiO}_{2}$ легко формируется в аморфном, изотропном состоянии и не кристаллизуется до весьма высоких
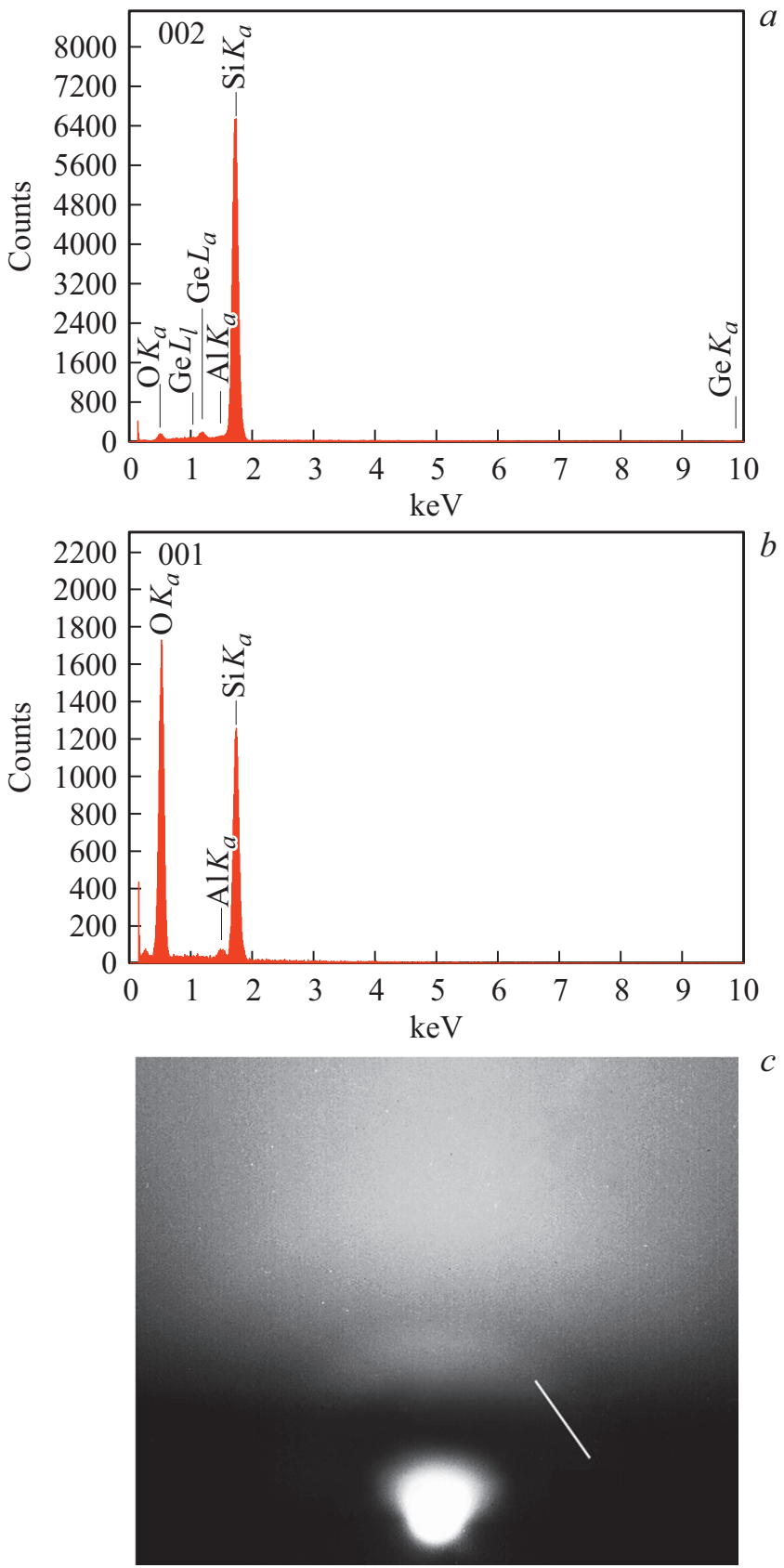

Рис. 1. Сравнение элементного состава поверхностного слоя диоксида кремния на боковой поверхности НHК $\mathrm{Si}(a)$ и кварца $(b)$, а также электронограмма, характеризующая аморфное состояние $\mathrm{SiO}_{2}(c)$.

температур (1473-1573 K) [12]. Видимо, поверхностные слои $\mathrm{SiO}_{x}$ при температурах < $1473 \mathrm{~K}$ могут формироваться на НHК Si по двухстадийной схеме: вначале осевой рост $\mathrm{HHK} \mathrm{Si}$, а затем радиальный рост оксидного слоя за счет окисления Si. При этом более тонкие ННК окисляются сильнее, чем более толстые. Образцы ННК, обработанные в НF и помещенные в вакуум на сутки, при повторном измерении не обнаруживают образования оксидного слоя. 

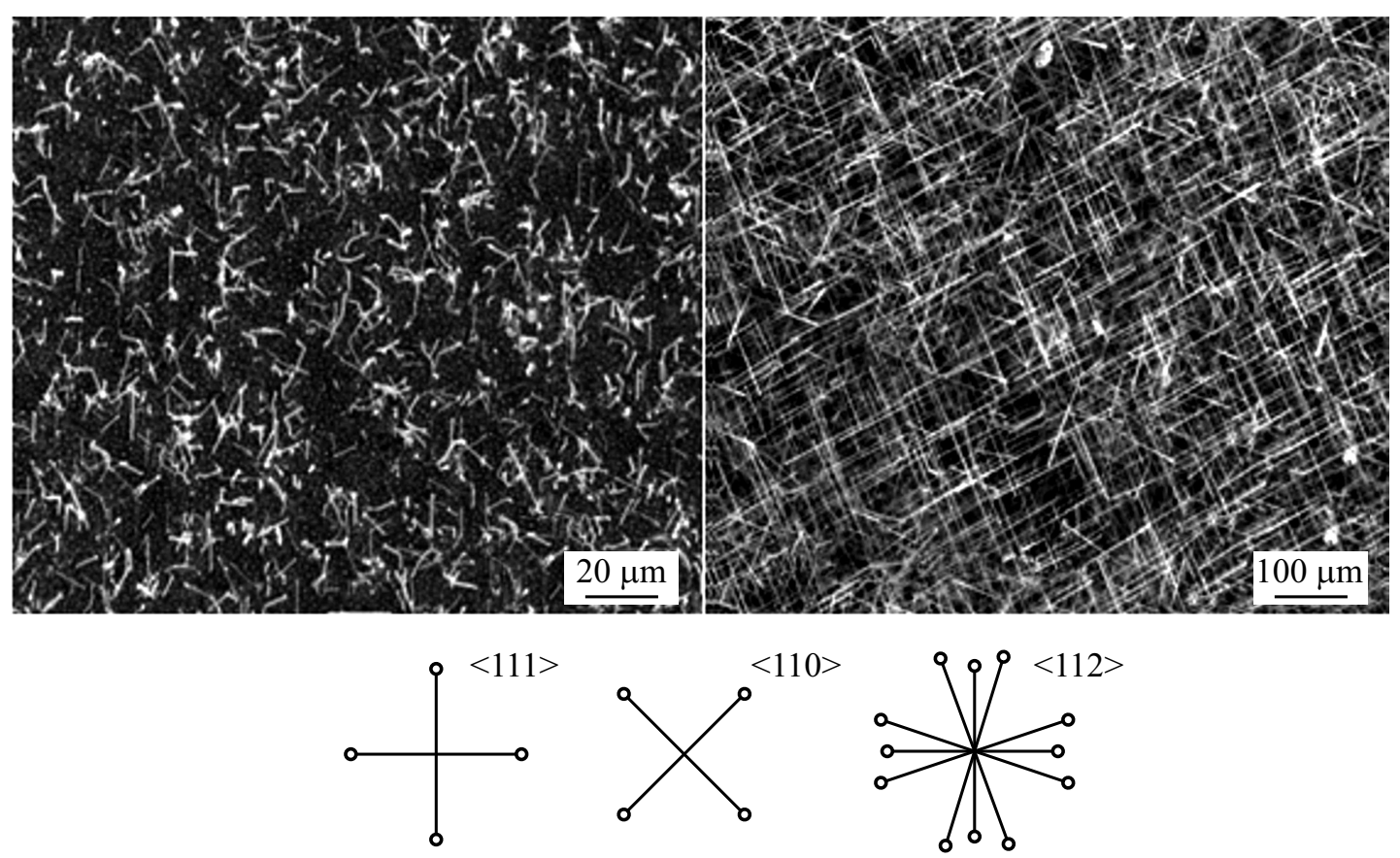

Рис. 2. Вид сверху в сканирующем электронном микроскопе $\mathrm{HHK} \mathrm{Si,} \mathrm{выращенных} \mathrm{на} \mathrm{кремниевой} \mathrm{подложке} \mathrm{ориентации}\{100\}$ : слева в потоке $\mathrm{H}_{2}$, не подвергшемся дополнительной очистке от присутствия $\mathrm{O}_{2}$ и паров воды, и справа в очищенном и осушенном потоке.

Из-за присутствия оксидного поверхностного слоя рост ННК Si заторможен, кристаллы характеризуются сильной морфологической неустойчивостью, имеют множественные изгибы, причем изгибы имеют одно- и многоколенный характер, а угол наклона при изгибах принимает различные значения, не зависящие ни от радиуса ННК, ни от типа металла-катализатора (рис. 2). Более толстые ННК имеют преимущественную ориентацию оси роста $\langle 111\rangle$, более тонкие - ориентированы хаотично в преимущественном направлении $\langle 110\rangle$. На окисленной $\mathrm{Si}$-подложке рост ННК либо полностью подавляется, либо наблюдается сферолитный рост множества мелких кристаллов. Неблагоприятное влияние оксидного поверхностного слоя на рост ННК наиболее сильно сказывается при низких температурах выращивания $(<1300 \mathrm{~K})$, а при высоких температурах $(>1400 \mathrm{~K})$ рост более устойчив.

Из-за окисления боковой поверхности ННК (при невысокой степени очистки водорода и присутствия в нем кислородосодержащих компонентов и паров воды) проволоки остаются цилиндрическими и не наблюдается огранения кристаллов по пар $\rightarrow$ кристалл (ПК)-механизму, характерного для двухстадийного процесса роста ННК $\mathrm{Si}$ в стерильных условиях (см. вставку на рис. 3,a) [11]. ПК-механизм относится к стадии радиального разрастания ННК за счет встраивания кристаллизуемого материала в ступени на боковой поверхности в процессе осевого роста по ПЖК механизму. Обычно ПК-механизм приводит к огранке $\mathrm{Si-кристалла,} \mathrm{растущего} \mathrm{в} \mathrm{направлении} \mathrm{\langle 111 \rangle} \mathrm{граня-}$ ми $\{112\}$ и $\{110\}$. Но, очевидно, при образовании оксидного поверхностного слоя ПК-рост блокируется, и ННК сохраняют цилиндрическую форму.

\section{1. Термодинамическая неустойчивость нитевидных нанокристаллов $\mathrm{Si}$ к окислению}

Непосредственно после выращивания образующиеся на боковой поверхности ННК слои $\mathrm{SiO}_{x}$ состоят из силоксановых цепей $\mathrm{Si}-\mathrm{O}-\mathrm{Si}-\mathrm{O}$ [14]. Поверхность $\mathrm{SiO}_{x}$ может содержать также силанольные группы $\mathrm{Si}-\mathrm{OH}$ [14]. Однако при температуре > 383-423 K начинается обратимая реакция превращения силанола в силоксан с выделением воды. Это сокращает длину связи $\mathrm{Si}-\mathrm{O}$ и существенно упрочняет ее. Данный процесс продолжается до тех пор, пока весь захваченный водород не будет удален с границы раздела. Поэтому для упрощения далее будем вести термодинамическое рассмотрение процессов образования $\mathrm{SiO}_{2}$, исходя из присутствия $\mathrm{O}_{2}$ в сухой газовой атмосфере.

При выращивании ННК $\mathrm{Si}$ в условиях наличия остаточных концентраций $\mathrm{O}_{2}$ в составе ростовой атмосферы $\mathrm{SiO}_{2}$ образуется по экзотермической реакции

$$
\mathrm{Si}_{(\mathrm{S})}+\mathrm{O}_{2(\mathrm{G})} \leftrightarrow \mathrm{SiO}_{2(\mathrm{~S})} ; \quad \Delta H_{1}<0,
$$

где $\Delta H_{1}$ - тепловой эффект реакции.

Рассмотрим простейший случай отсутствия растворов между твердыми веществами, т.е. когда $\mathrm{Si}$ и его ок- 
$a$

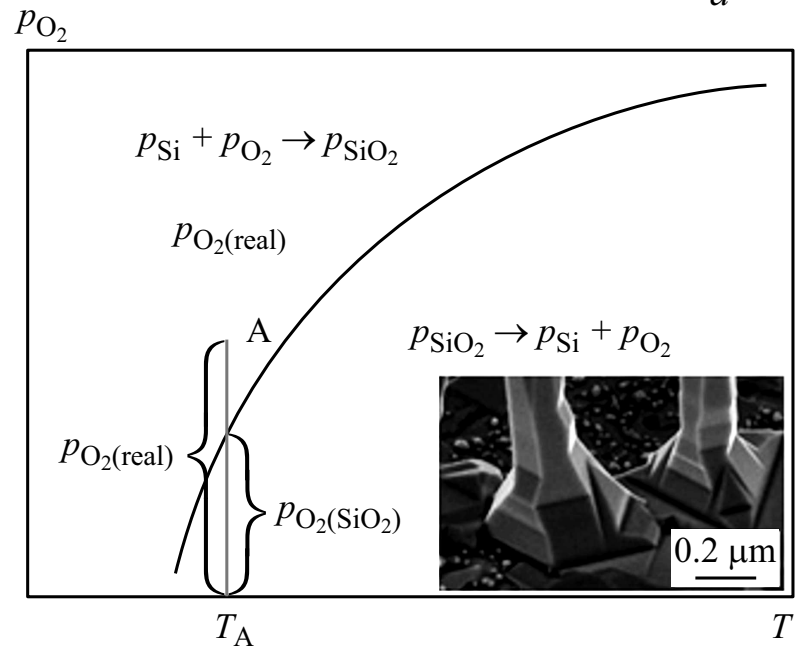

$p_{\mathrm{O}_{2}}$

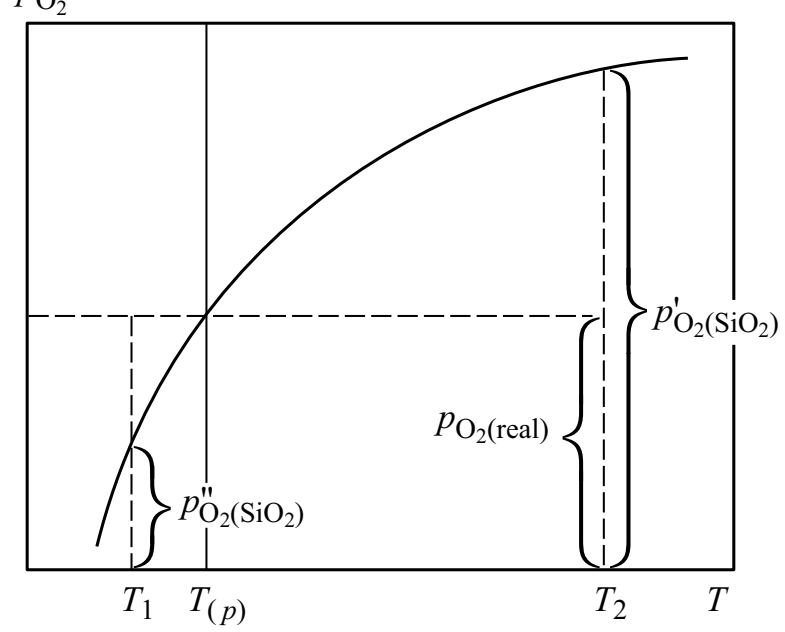

Рис. 3. График для определения направления реакции образования или диссоциации $\mathrm{SiO}_{2}: a-$ при любом парциальном давлении $\mathrm{O}_{2}, b-$ при заданном давлении.

сид представляют собой самостоятельные чистые фазы, общее число которых, включая и газообразную, равно трем. В этом случае рассматриваемая система двухкомпонентна - три вещества и одно уравнение реакции. В соответствии с правилом фаз Гиббса система одновариантна $(C=2+2-3=1)$. Одновариантность системы означает, что равновесное давление $\mathrm{O}_{2}$ зависит только от температуры $p_{\mathrm{O}_{2}}=f(T)$.

Вид данной функции можно получить из выражения для константы равновесия реакции (1)

$$
K=a_{\mathrm{SiO}_{2}} /\left(a_{\mathrm{Si}} \cdot p_{\mathrm{O}_{2}}\right),
$$

где $a_{\mathrm{SiO}_{2}}$ и $a_{\mathrm{Si}}-$ активности $\mathrm{SiO}_{2}$ и $\mathrm{Si}$ соответственно.

Поскольку активности чистых твердых веществ в макроскопическом состоянии практически равны единице, то из (2) получаем

$$
K=1 / p_{\mathrm{O}_{2}}=f(T) .
$$

Из (3), обозначив $p_{\mathrm{O}_{2}}$ как $p_{\mathrm{O}_{2}\left(\mathrm{SiO}_{2}\right)}$, найдем

$$
p_{\mathrm{O}_{2}\left(\mathrm{SiO}_{2}\right)}=1 / K=f^{-1}(T) \text {. }
$$

На основании выражения (4) по аналогии с оксидами металлов равновесное парциальное давление кислорода $p_{\mathrm{O}_{2}\left(\mathrm{SiO}_{2}\right)}$ в реакции образования или диссоциации диоксида кремния можно назвать упругостью диссоциации $\mathrm{SiO}_{2}$.

С учетом (4) запишем выражение для стандартного изменения изобарного потенциала $\left(-\Delta G^{0}=R T \ln K\right)$,

$$
-\Delta G^{0}=-R T \ln p_{\mathrm{O}_{2}\left(\mathrm{SiO}_{2}\right)} .
$$

В соответствии с $(5)$, чем больше $\left(-\Delta G^{0}\right)$ и чем меньше $p_{\mathrm{O}_{2}\left(\mathrm{SiO}_{2}\right)}$, тем больше сродство $\mathrm{Si}$ к $\mathrm{O}_{2}$, тем прочнее и устойчивее оксид. С понижением устой- чивости $\mathrm{SiO}_{2}$ способен полнее диссоциировать при данной температуре, и парциальное давление $\mathrm{O}_{2}$ при достижении равновесия возрастает. При повышении $T$ константа равновесия $\mathrm{K}$ рассматриваемой экзотермической реакции убывает, упругость диссоциации по уравнению (4) растет, т.е. устойчивость оксида уменьшается.

Для оценки направленности протекания процесса воспользуемся уравнением изотермы химической реакции Вант-Гоффа [15], которое применительно к рассматриваемой нами системе будет иметь вид

$$
\begin{aligned}
\Delta G & =R T\left(\ln \left(1 / p_{\mathrm{O}_{2}(\text { real })}\right)-\ln \left(1 / p_{\mathrm{O}_{2}\left(\mathrm{SiO}_{2}\right)}\right)\right) \\
& =R T\left(\ln p_{\mathrm{O}_{2}\left(\mathrm{SiO}_{2}\right)}-\ln p_{\mathrm{O}_{2}(\text { real })}\right) .
\end{aligned}
$$

Константа равновесия К по уравнению (3) выражается в данном случае через упругость диссоциации $\mathrm{SiO}_{2}$ как $p_{\mathrm{O}_{2}\left(\mathrm{SiO}_{2}\right)}$. Следовательно, произведение фактических концентраций строится также, как и константа равновесия, а $p_{\mathrm{O}_{2} \text { (real) }}$ означает фактическое парциальное давление $\mathrm{O}_{2}$ в газовой среде, окружающей $\mathrm{SiO}_{2}$ и $\mathrm{Si}$.

Таким образом, знак $\Delta G$, а отсюда и возможное направление процесса при заданной температуре, определяется соотношением значений величин $p_{\mathrm{O}_{2}\left(\mathrm{SiO}_{2}\right)}$ и $p_{\mathrm{O}_{2} \text { (real) }}$. Первая из них отражает свойства $\mathrm{SiO}_{2}$ и зависит только от температуры, а вторая характеризует состав среды газовой фазы и никак не связана с температурой. Между указанными величинами возможны следующие соотношения:

1. $p_{\mathrm{O}_{2} \text { (real) }}>p_{\mathrm{O}_{2}\left(\mathrm{SiO}_{2}\right)}$, т.е. в системе имеется избыток $\mathrm{O}_{2}$ в сравнении с равновесной концентрацией. При этом $\Delta G<0$ и реакция может идти в сторону образования $\mathrm{SiO}_{2}$. 
2. $p_{\mathrm{O}_{2}(\text { real })}<p_{\mathrm{O}_{2}\left(\mathrm{SiO}_{2}\right)}$, фактическое давление $\mathrm{O}_{2}$ меньше равновесного. В этом случае $\Delta G>0$ и процесс может идти в сторону диссоциации $\mathrm{SiO}_{2}$.

3. При $p_{\mathrm{O}_{2} \text { (real) }}=p_{\mathrm{O}_{2}\left(\mathrm{SiO}_{2}\right)}, \Delta G=0$ и система находится в равновесии.

Изложенное проиллюстрировано на рис. 3, $а$. Графики рис. 3 построены по уравнению [16]

$$
\ln p_{\mathrm{O}_{2}}=\left(\Delta H_{T}^{0} / 4.576 T\right)-\left(\Delta S_{T}^{0} / 4.576\right),
$$

где $\Delta H_{T}^{0}$ и $\Delta S_{T}^{0}-$ изменения энтальпии и энтропии при данной температуре соответственно, 4.576 - числовой коэффициент, устанавливаемый из опытных данных для определенного температурного интервала, $T$ - абсолютная температура.

Вид функции $p_{\mathrm{O}_{2}}=f(T)$ (7) можно получить интегрированием уравнения изобары химической реакции (1) [14]. Точки, расположенные на кривой рис. 3, соответствуют равновесным системам и характеризуют упругость диссоциации $\mathrm{SiO}_{2}$, которая возрастает с повышением температуры. Область графика выше кривой (например, точка A) соответствует произвольным неравновесным системам с давлениями $p_{\mathrm{O}_{2}(\text { real })}>p_{\mathrm{O}_{2}\left(\mathrm{SiO}_{2}\right)}$, в которых возможно окисление. Поле ниже кривой отвечает условию $p_{\mathrm{O}_{2} \text { (real) }}<p_{\mathrm{O}_{2}\left(\mathrm{SiO}_{2}\right)}$, и здесь возможна лишь диссоциация оксида и рост ННК чистого $\mathrm{Si}$.

Таким образом, с помощью графика $p_{\mathrm{O}_{2}}=f(T)$ для каждого типа НHК (Si или др.) можно находить температуры и состав газовой фазы, при которых возможны образование или диссоциация диоксида. По выражению (7) можно рассчитать температуру $T_{0}$ начала реакции разложения $\mathrm{SiO}_{2}$ при заданной концентрации $\mathrm{O}_{2}$.

Для определения направления протекания процесса в газовой фазе постоянного состава необходимо принять $p_{\mathrm{O}_{2} \text { (real) }}=$ const. На графике данное условие будет отображаться прямой, параллельной оси абсцисс (рис. $3, b$ ).

Из рис. $3, b$ видно, что равновесие системы или условие $p_{\mathrm{O}_{2}(\text { real })}=p_{\mathrm{O}_{2}\left(\mathrm{SiO}_{2}\right)}=\mathrm{const}$ осуществимо лишь при одной температуре $T_{p}$. При любых других температурах равновесие невозможно. Если $T_{1}<T_{p}$, то $p_{\mathrm{O}_{2} \text { (real) }}>p_{\mathrm{O}_{2}\left(\mathrm{SiO}_{2}\right)}^{\prime \prime}$, и реакция протекает только в сторону образования оксида. При $T_{2}>T_{p} \quad\left(p_{\mathrm{O}_{2}(\text { real })}<p_{\mathrm{O}_{2}\left(\mathrm{SiO}_{2}\right)}^{\prime}\right)$ процесс должен идти в противоположном направлении. Известно, например, что в атмосфере воздуха при низкой, комнатной температуре на чистой поверхности $\mathrm{Si}$ слой $\mathrm{SiO}_{2}$ толщиной 1-1.5 нм образуется практически мгновенно и в дальнейшем растет со скоростью $\sim 1 \mathrm{HM} /$ сут [17].

Таким образом, $T_{p}$ есть та температура, выше которой протекает только диссоциация, т.е. это температура начала разложения $\mathrm{SiO}_{2}$ в газовой фазе с постоянным парциальным давлением $\mathrm{O}_{2}$. Она тем выше, чем больше $p_{\mathrm{O}_{2} \text { (real) }}$ в газе.

Логарифм упругости диссоциации для реакции окисления $\mathrm{Si}$ составляет: при $T=1000 \mathrm{~K} \lg p_{\mathrm{O}_{2}\left(\mathrm{SiO}_{2}\right)}=-36.1$, при $T=1600 \mathrm{~K} \lg p_{\mathrm{O}_{2}\left(\mathrm{SiO}_{2}\right)}=-19.0$ [18]. Указанные значения величин свидетельствуют о высоком сродстве
$\mathrm{Si}$ к $\mathrm{O}_{2}$ и практической необратимости реакции образования $\mathrm{SiO}_{2}$ (константа равновесия $\mathrm{K}$ существенно больше единицы, а $\left.p_{\mathrm{O}_{2}\left(\mathrm{SiO}_{2}\right)} \ll 1 \mathrm{aTM}\right)$. Это означает, что при температурах синтеза 500- $1400 \mathrm{~K}$ HНК Si термодинамически неустойчивы в газовой фазе, содержащей любые ощутимо малые концентрации $\mathrm{O}_{2}$, и при благоприятных кинетических условиях $\mathrm{Si}$ должен всецело превращаться в диоксид. Термическая диссоциация $\mathrm{SiO}_{2}$ в условиях роста ННК $\mathrm{Si}$ практически неосуществима. Для ее осуществления необходимо либо понизить давление кислорода до чрезвычайно малых, недостигаемых значений, либо поднять температуру до такой, которую нельзя получить в обычных условиях.

Полагая для реакции (1)

$$
\left.\Delta H_{T}^{0}=-872.41 \text { кДж/(моль } \mathrm{O}_{2}\right)
$$

и

$$
\left.\Delta S_{T}^{0}=-181.71 \text { Дж/(град · моль } \mathrm{O}_{2}\right)
$$

и концентрацию $\mathrm{O}_{2}$ в газовой фазе $0.03 \%$ [16], по формуле (7) найдем температуру $T_{0}$ начала разложения $\mathrm{SiO}_{2}$. При общем давлении в системе в 1 атм и $p_{\mathrm{O}_{2} \text { (real) }}=3 \cdot 10^{-4}$ атм получаем $T_{0} \approx 3000 \mathrm{~K}$. При этом диссоциация $\mathrm{SiO}_{2}$ в атмосфере воздуха, содержащего $21 \% \mathrm{O}_{2}\left(p_{\mathrm{O}_{2} \text { (real) }}=0.21\right.$ атм $)$, и суммарном давлении $P_{\Sigma}=1$ атм становится возможной лишь при температуpe $>4000 \mathrm{~K}$.

Таким образом, становится понятным, что в экспериментальных условиях выращивания ННК Si с использованием неочищенного $\mathrm{H}_{2}$, содержащего $1 \cdot 10^{-3} \% \mathrm{O}_{2}$, при температуре $<1400 \mathrm{~K}$ всегда должен образовываться диоксид $\mathrm{SiO}_{2}$, а единственная возможность препятствовать этому процессу — очищать газовую фазу от присутствия $\mathrm{O}_{2}$.

\section{2. Влияние степени дисперсности нитевидных нанокристаллов на условия образования $\mathrm{SiO}_{2}$}

Энергетическое состояние частиц вещества твердой фазы (для $\mathrm{SiO}_{2}$ это ионы $\mathrm{Si}^{4+}$ и $\mathrm{O}_{2}^{4-}$ ), расположенных на внешней поверхности ННК и внутри кристалла, неодинаково. Поверхностные атомы и ионы подвержены несиммеричным межчастичным взаимодействиям, вследствие чего у них возникает избыточная поверхностная энергия в сравнении с частицами в объеме. Поэтому среднее значение изобарного потенциала поверхностных атомов и ионов больше, чем у частиц в объеме. Поскольку в зависимости от поперечного размера ННК наблюдаются различия в устойчивости кристаллов к окислению, рассмотрим влияние характера кристаллического строения $\mathrm{Si}$ и $\mathrm{SiO}_{2}$ на упругость диссоциации.

С повышением степени дисперсности (в нашем случае с уменьшением размеров ННК) величина изобарного потенциала для определенного количества вещества возрастает, так как при этом увеличивается доля частиц, 
расположенных на поверхности. Поскольку активности можно определить из уравнения

$$
G=G^{0}+R T \ln a,
$$

$G$ и $G^{0}$ - значения мольных изобарных потенциалов вещества в данном и стандартном состояниях при одной и той же температуре, примем в качестве стандартного состояния $\mathrm{Si}$ и $\mathrm{SiO}_{2}$ такое состояние, при котором эти вещества чистые и представлены макроскопическими кристаллами, $a_{\mathrm{SiO}_{2}}=1$ и $a_{\mathrm{Si}}=1$. Но при учете поверхностных явлений для нанокристаллов нельзя принимать активности $a_{\mathrm{SiO}_{2}}$ и $a_{\mathrm{Si}}$ равными единице. Если $G$ отнести к нанодисперсному состоянию НHК $\mathrm{Si}$, то в соответствии с вышесказанным $G>G^{0}$ и $G-G^{0}=R T \ln a_{\mathrm{Si}}>0$. Следовательно, для ННК в нанодисперсном состоянии $\ln a_{\mathrm{Si}}>0$ и $a_{\mathrm{Si}}>1$. Отсюда следует, что чем выше дисперсность, т.е. меньше диаметр ННК, тем больше активность $a_{\mathrm{Si}}$.

Выразим из уравнения (2) равновесное давление $\mathrm{O}_{2}$

$$
p_{\mathrm{O}_{2}\left(\mathrm{SiO}_{2}\right)}=(1 / K)\left(a_{\mathrm{SiO}_{2}} / a_{\mathrm{Si}}\right) .
$$

Поскольку константа равновесия, выраженная через активности $\mathrm{Si}$ и $\mathrm{SiO}_{2}$, однозначно определяется температурой, то при $T=$ const можно считать и $\mathrm{K}=$ const. Отсюда при постоянной температуре увеличение степени дисперсности $\mathrm{SiO}_{2}$, а значит и возрастание $a_{\mathrm{SiO}_{2}}$, в соответствии с (9) ведет к росту упругости диссоциации, т.е. к разложению диоксида, а повышение дисперсности $\mathrm{Si}$ (в нашем случае уменьшение размеров HНК $\mathrm{Si}$ ) и $a_{\mathrm{Si}}-$ к ее уменьшению, т. е. окислению $\mathrm{Si}$ и образованию оксидных слоев ННК. Видим, что $p_{\mathrm{O}_{2}\left(\mathrm{SiO}_{2}\right)}$ перестает быть функцией только температуры, а зависит еще от характера кристаллической структуры твердых фаз, в частности от поперечных размеров ННК.

Физически это можно пояснить следующим образом. Изобарный потенциал G (или химический потенциал) характеризует стремление компонента к выходу из той фазы, в которой он находится. Чем выше дисперсность вещества, т.е. меньше размеры частиц, тем у него больше $\mathrm{G}$, и тем оно менее устойчиво, а следовательно, и более активно. Если сравнивать крупнокристаллический $\mathrm{SiO}_{2}$ с нанокристаллическим, то во втором случае вещество менее устойчиво, у него выше стремление к распаду, а следовательно, и больше упругость диссоциации. Напротив, увеличение степени дисперсности (уменьшение поперечных размеров) НHК Si приводит к тому, что у них повышается тенденция к взаимодействию с $\mathrm{O}_{2}$, последний прочнее удерживается кремнием, и упругость диссоциации падает.

Полученные результаты указывают на то, сколь тщательно должна быть проведена очистка газовой фазы от присутствия $\mathrm{O}_{2}$ при выращивании НHК $\mathrm{Si}$. На начальном этапе окисления, когда образующиеся на поверхности ННК зародыши и кластеры $\mathrm{SiO}_{2}$ имеют субнаноскопические размеры, образование оксидной фазы затруднено. Но после возникновения сплошного оксидного слоя фаза
$\mathrm{SiO}_{2}$ становится более устойчивой. При этом более тонкие ННК должны окисляться сильнее, чем более толстые.

\section{3. Практическая неосуществимость водородного восстановления и термической диссоциации образующегося оксида}

Если в газофазной атмосфере присутствуют газообразные реагенты, способные восстанавливать твердый $\mathrm{SiO}_{2}$, например, $\mathrm{H}_{2}$ при получении ННК водородным восстановлением галогенидов кремния [19], то параллельно с процессом окисления ННК $\mathrm{Si}$ может идти гетерогенная реакция восстановления образующегося оксида. Рассмотрим данный процесс на примере эндотермической реакции

$$
\mathrm{SiO}_{2(\mathrm{~S})}+2 \mathrm{H}_{2(\mathrm{G})} \leftrightarrow \mathrm{Si}_{(\mathrm{S})}+2 \mathrm{H}_{2} \mathrm{O}_{(\mathrm{G})} ; \quad \Delta \mathrm{H}_{2}>0 .
$$

Тепловой эффект эндотермической реакции водородного восстановления $\mathrm{SiO}_{2}$ (10) меньше теплового эффекта эндотермической реакции термической диссоциации оксида (обратная реакция по уравнению (1)) на величину экзотермического эффекта реакции образования паров воды из элементов:

$$
\mathrm{O}_{2(\mathrm{G})}+2 \mathrm{H}_{2(\mathrm{G})} \leftrightarrow 2 \mathrm{H}_{2} \mathrm{O}_{(\mathrm{G})} ; \quad \Delta H_{\mathrm{C}}<0
$$

Это означает, что водородное восстановление $\mathrm{SiO}_{2}$, требующее меньших энергетических затрат $\left(\Delta H_{C} \approx-484.4\right.$ кДж), может протекать при более низкой температуре, чем термическая диссоциация диоксида, что является значительным преимуществом.

Приняв активности чистого $\mathrm{Si}$ и его диоксида в макроскопическом состоянии равными единице, получим выражение для константы равновесия реакции (10)

$$
K_{1}=p_{\mathrm{H}_{2} \mathrm{O}}^{2} / p_{\mathrm{H}_{2}}^{2}=\left(\% \mathrm{H}_{2} \mathrm{O}\right)_{(\mathrm{ecv})}^{2} /\left(\% \mathrm{H}_{2}\right)_{(\mathrm{ecv})}^{2},
$$

где $p_{\mathrm{H}_{2} \mathrm{O}}, p_{\mathrm{H}_{2}}, \% \mathrm{H}_{2} \mathrm{O}_{(\text {ecv })}$ и $\% \mathrm{H}_{2(\text { ecv })}$ - равновесные парциальные давления и процентные концентрации паров воды и водорода в газовой фазе.

Из (12) в соответствии с выводами правила фаз равновесные концентрации $\mathrm{H}_{2}$ и $\mathrm{H}_{2} \mathrm{O}$ зависят только от температуры. Поскольку реакция (10) протекает с поглощением тепла, то $\mathrm{K}_{1}$ возрастает, а \% $\mathrm{H}_{2(\mathrm{ecv})}^{2}$ убывает с повышением температуры (рис. 4).

Уравнение изотермы реакции для реакции (10) имеет вид

$$
\Delta G_{1}=R T\left(\ln \left(\left(\% \mathrm{H}_{2} \mathrm{O}\right)_{(\text {real })}^{2} /\left(\% \mathrm{H}_{2}\right)_{(\text {real })}^{2}\right)-\ln K_{1}\right),
$$

где $\Delta G_{1}-$ изменение изобарного потенциала реакции $(10),\left(\% \mathrm{H}_{2} \mathrm{O}\right)_{(\text {real })}^{2} /\left(\% \mathrm{H}_{2}\right)_{(\text {real })}^{2}-$ соотношение фактических концентраций веществ.

Из (13) следует, что при данной температуре направление процесса водородного восстановления и сродство $\mathrm{SiO}_{2}$ к $\mathrm{H}_{2}$ (определяемые знаком и величиной ) зависят 


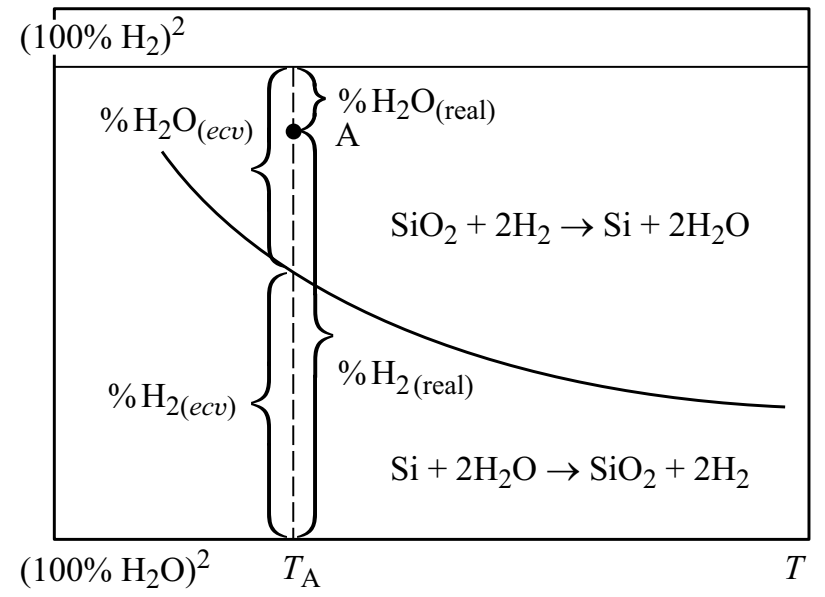

Рис. 4. Зависимость равновесного состава газовой фазы от температуры в эндотермической реакции восстановления $\mathrm{Si}$ водородом.

от соотношения фактических концентраций $\mathrm{H}_{2}$ и $\mathrm{H}_{2} \mathrm{O}$. Если $\left(\% \mathrm{H}_{2} \mathrm{O}\right)_{(\text {real) }}^{2} /\left(\% \mathrm{H}_{2}\right)_{(\text {real })}^{2}<\left(\% \mathrm{H}_{2} \mathrm{O}\right)_{(\text {ecv) }}^{2} /\left(\% \mathrm{H}_{2}\right)_{(\text {ecv) }}^{2}$, то $\Delta G_{1}<0$, и смесь данного состава будет восстанавливать $\mathrm{Si}$, в противоположном случае - будет окислять $\mathrm{Si}$. Сказанное проиллюстрировано графиком рис. 4.

Точки на кривой отвечают равновесным условиям или нейтральным газовым смесям $\mathrm{H}_{2}$ и $\mathrm{H}_{2} \mathrm{O}$ как по отношению к $\mathrm{SiO}_{2}$, так и к $\mathrm{Si}$. Точки выше кривой (например, точка $A$ ) соответствуют избытку $\mathrm{H}_{2}$ и недостатку $\mathrm{H}_{2} \mathrm{O}$ в сравнении с равновесными концентрациями, т.е. восстановительным газовым смесям, так как здесь $\Delta G_{1}<0$. Ниже кривой располагаются точки, принадлежащие окислительным по отношению к $\mathrm{Si}$ смесям, здесь $\Delta G_{1}>0$.

Таким образом, верхнее поле является областью температур и составов газа, в которой устойчив $\mathrm{Si}$; в нижней области устойчив $\mathrm{SiO}_{2}$.

Равновесие реакции (10) определяется равенством $p_{\mathrm{O}_{2}\left(\mathrm{SiO}_{2}\right)}=p_{\mathrm{O}_{2}\left(\mathrm{H}_{2} \mathrm{O}\right)}$, где $p_{\mathrm{O}_{2}\left(\mathrm{H}_{2} \mathrm{O}\right)}=\left(1 / K_{2}\right)\left(\left(\% \mathrm{H}_{2} \mathrm{O}\right)_{(\text {real })}^{2} /\right.$ $\left.\left(\% \mathrm{H}_{2}\right)_{(\text {real })}^{2}\right)^{2}-$ равновесное парциальное давление $\mathrm{O}_{2}$ в реакции (11), характеризующее окислительную способность газовой фазы. Варьируя соотношение между $\% \mathrm{H}_{2} \mathrm{O}$ и $\% \mathrm{H}_{2}$, можно подобрать для $\mathrm{SiO}_{2}$ равновесную газовую смесь. При данной температуре повышение прочности диоксида (уменьшение его упругости диссоциации $p_{\mathrm{O}_{2}\left(\mathrm{SiO}_{2}\right)}$ требует соответствующего снижения и $p_{\mathrm{O}_{2}\left(\mathrm{H}_{2} \mathrm{O}\right)}$ (окислительной способности), т.е. изменения равновесного состава газовой фазы в направлении уменьшения концентрации окислителя $\left(\mathrm{H}_{2} \mathrm{O}\right)$ и увеличения концентрации восстановителя $\left(\mathrm{H}_{2}\right)$. Другими словами, чем прочнее диоксид, тем богаче восстановителем $\left(\mathrm{H}_{2}\right)$ должен быть газ, или тем выше располагаются равновесные кривые на графике в координатах $\% \mathrm{H}_{2}-T$. Именно по этой причине при выращивании $\mathrm{HHK} \mathrm{Si}$ в потоке $\mathrm{H}_{2}$, не подвергшемся дополнительной очистке от остатков $\mathrm{O}_{2}$, и в котором присутствуют следовые остатки паров воды, на поверхности нанокристаллов и ростовой подложке образуются слои $\mathrm{SiO}_{2}$.

При заданной концентрации $\mathrm{H}_{2}$ в атмосфере увеличение температуры $T$ процесса будет способствовать восстановительной способности газовой фазы. Последний вывод объясняет наблюдаемые экспериментальные результаты, когда влияние оксидного поверхностного слоя наиболее сильно сказывается при низких температурах $(<1300 \mathrm{~K})$, а при высоких $T$ (1400 К и выше) рост ННК более устойчив.

Произведем оценку минимального расхода $\mathrm{H}_{2}$ на восстановление окислов кремния. Представим замкнутый сосуд, в котором находится 1 моль оксида $\mathrm{SiO}_{2}$. Найдем, сколько нужно ввести в него $\mathrm{H}_{2}$ для того, чтобы обеспечить полное восстановление оксида при данной температуре. В результате реакции (10) в сосуде будет накапливаться $\mathrm{H}_{2} \mathrm{O}$, а количество $\mathrm{H}_{2}$ - уменьшаться. Процесс прекратится тогда, когда газовая смесь достигнет равновесного состава.

На восстановление 1 моля $\mathrm{SiO}_{2}$ по реакции (10) требуется 2 моля $\mathrm{H}_{2}$. Однако в сосуд необходимо ввести $n>2$ молей $\mathrm{H}_{2}$ для нейтрализации образующейся $\mathrm{H}_{2} \mathrm{O}$, что выразится уравнением

$$
\mathrm{SiO}_{2(\mathrm{~S})}+n \mathrm{H}_{2(\mathrm{G})} \rightarrow \mathrm{Si}_{(\mathrm{S})}+2 \mathrm{H}_{2} \mathrm{O}_{(\mathrm{G})}+(n-2) \mathrm{H}_{2(\mathrm{G})} .
$$

Величина $n$ будет минимальной в том случае, если конечная смесь из 1 моля $\mathrm{H}_{2} \mathrm{O}$ и $(n-2)$ молей $\mathrm{H}_{2}$ будет равновесной, т.е. когда $\frac{1}{n-2}=K_{1}$. Здесь $K_{1}-$ константа равновесия для реакции (10). Для реакции (10) $K_{1}$ определяется выражением (12). Отсюда $n_{\min }\left(\mathrm{SiO}_{2}\right)=2+\left(1 / K_{1}\right)$.

Если количество молей $\mathrm{H}_{2}$ будет меньше $n_{\min }\left(\mathrm{SiO}_{2}\right)$, то произойдет лишь частичное восстановление кремния. Для трудно восстановимого $\mathrm{SiO}_{2} K_{1} \ll 1$ и равновесный газ близок к $100 \% \mathrm{H}_{2} / \mathrm{H}_{2} \mathrm{O}$. Поэтому необходимый расход $\mathrm{H}_{2}$ становится настолько большим, что при выращивании ННК восстановительный процесс теряет практическое значение. Другими словами, для восстановления $\mathrm{Si}$ из $\mathrm{SiO}_{2}$ при температуре $1000 \mathrm{~K}$ необходимо все время поддерживать содержание паров $\mathrm{H}_{2} \mathrm{O}$ в газовой фазе существенно $<10^{-6} \%$, что невозможно обеспечить современными методами осушки водорода. Поэтому рост HНК Si будет затруднен.

\section{4. Влияние поверхностного оксидного слоя на рост нитевидных нанокристаллов}

Образующиеся поверхностные оксидные слои могут влиять как на зарождение, так и на рост НHК Si. Слой $\mathrm{SiO}_{2}$ расположен между двумя средами: кристаллом или подложкой $\mathrm{Si}$ и газом. В пределах слоя концентрация кислорода должна уменьшаться к границе $\mathrm{SiO}_{2} \mid \mathrm{Si}$, а концентрация кремния - в противоположном направлении. Это создает предпосылки для диффузии веществ в слое $\mathrm{SiO}_{2}$. Коэффициент диффузии в $\mathrm{SiO}_{2}$ будет зависеть от макростроения оксида, определяемого соотношением 
мольных объемов диоксида $\left(V_{\mathrm{SiO}_{2}}\right)$ и кремния $\left(V_{\mathrm{Si}}\right)$. Поскольку мольный объем $\mathrm{Si}$ меньше мольного объема оксида $\left(V_{\mathrm{Si}} / V_{\mathrm{SiO}_{2}}\right) \approx 0.585$, можно считать, что $\mathrm{SiO}_{2}$ покрывает ННК и ростовую подложку сплошным плотным слоем, создающим большое диффузионное сопротивление и затрудняющим окисление. Отсюда можно предположить, что процесс образования $\mathrm{SiO}_{2}$ лимитируется внутренней диффузией.

Сплошной окисный слой является препятствием для растворения $\mathrm{Si}$ в жидкофазных частицах $\mathrm{Au}-$ катализатора еще на этапе их сплавления с ростовой подложкой. Это может приводить к неустойчивости фронта кристаллизации под каплей на начальной стадии роста ННК. В результате поверхность раздела кристалл/жидкость перестает быть плоской по всей площади фронта, что может привести к частичному захвату жидкого сплава в кристалл. Такой процесс вызывает образование дислокаций, трещин и полей напряжений в ННК и приводит к разбиению катализатора на отдельные капли и сферолитному росту множества кристаллов.

Окисление боковой поверхности ННК может влиять на величину контактного угла и равновесие капли катализатора на вершине кристалла, поскольку свободная поверхностная энергия границы $\mathrm{SiO}_{2} /$ газ существенно отличается от данной величины для межфазной границы $\mathrm{Si} /$ газ. По этой причине может возникать морфологическая неустойчивость ННК, представляющая собой изменение диаметра и образование изгибов кристаллов, а также захват двухкомпонентного сплава при случайном изменении направления роста. Структурные дефекты ННК являются эффективными концентраторами электрически активных примесей.

Поэтому понимание термодинамики образования поверхностных оксидных слоев и его влияния на рост $\mathrm{HHK} \mathrm{Si}$, полученное из нашего исследования, развивает имеющиеся представления о термодинамике образования оксидных поверхностных слоев и их влиянии на рост ННК $[12,16,20]$, что может быть решающим шагом в осуществлении воспроизводимого синтеза перспективных наноматериалов.

\section{4. Заключение}

Установлено, что при выращивании ННК Si в присутствии наночастиц $\mathrm{Au}$ в потоке $\mathrm{H}_{2}$, не подвергшемся дополнительной очистке от остатков $\mathrm{O}_{2}$ и паров воды, на поверхности нанопроволок и на ростовой подложке образуются слои $\mathrm{SiO}_{2}$. Из-за присутствия оксидного поверхностного слоя рост НHК Si заторможен, кристаллы характеризуются сильной морфологической неустойчивостью. Влияние оксидного поверхностного слоя наиболее сильно сказывается при низких температурах выращивания $(<1300 \mathrm{~K})$, а при высоких температурах (1400 К и выше) рост ННК более устойчив.

Показано, что при постоянной температуре увеличение степени дисперсности $\mathrm{Si}$, т.е. уменьшение попе- речных размеров ННК, приводит к тому, что у них повышается тенденция к взаимодействию с $\mathrm{O}_{2}$, и последний прочнее удерживается кремнием. При этом более тонкие ННК окисляются сильнее, чем более толстые. При заданной концентрации $\mathrm{H}_{2}$ в атмосфере увеличение температуры процесса будет усиливать восстановительную способность газовой фазы.

При температурах синтеза 750-1400 K HНК Si термодинамически неустойчивы в газовой фазе, содержащей любые ощутимо малые концентрации $\mathrm{O}_{2}$, и при благоприятных кинетических условиях кристаллы $\mathrm{Si}$ должны всецело превращаться в оксид. При этом необходимый расход $\mathrm{H}_{2}$ на водородное восстановление $\mathrm{SiO}_{2}$ является настолько большим, что при выращивании ННК восстановительный процесс теряет практическое значение. Термическая диссоциация $\mathrm{SiO}_{2}$ в условиях роста ННК $\mathrm{Si}$ также практически неосуществима. Следовательно, контроль за содержанием $\mathrm{O}_{2}$ в газовой фазе при выращивании $\mathrm{HHK} \mathrm{Si}$ является критически важным.

\section{Финансирование работы}

Исследование выполнено при финансовой поддержке Российского фонда фундаментальных исследований в рамках научного проекта № 19-33-90219 на оборудовании Центра коллективного пользования „Наноэлектроника и нанотехнологические приборы“ Воронежского государственного технического университета.

\section{Конфликт интересов}

Авторы заявляют, что у них нет конфликта интересов.

\section{Список литературы}

[1] F. Glas. Phys. Rev. B, 74, 121302 (2006).

[2] А.А. Корякин, С.А. Кукушкин, Н.В. Сибирев. ФТП, 53 (3), 370 (2019).

[3] И.П. Сошников, Г.Э. Цырлин, А.А.Тонких, Ю.Б. Самсоненко, В.Г. Дубровский, В.М. Устинов, О.М. Горбенко, D. Litvinov, D. Gerthsen. ФTT, 47 (12), 2121 (2005).

[4] B.J. Ohlsson, M.T. Björk, M.H. Magnusson, K. Deppert, L. Samuelson. Appl. Phys. Lett., 79 (20), 3335 (2001).

[5] S. Datta. Electronic Transport in Mesoscopic Systems (Cambridge University Press, 1997).

[6] Y. Arakawa, H. Sakaki. Appl. Phys. Lett., 40, 939 (1982).

[7] Патент RU № 2517924, В.А. Небольсин, А.И. Дунаев. Зарегистр. в Гос. реестре изобретений 11.04.2014 г.

[8] В.А. Небольсин, N. Swaikat, А.Ю. Воробьев. Письма ЖТФ, 44 (23), 16 (2018).

[9] S. Misra, L. Yu, W. Chen, M. Foldyna, P.R. Cabarrocas. J. Phys. D: Appl. Phys., 47 (39), 393001 (2014).

[10] A.I. Boukai, Y. Bunimovich, J. Tahir-Kheli, J.-K. Yu, W.A. Goddard, J.R. Heath. Nature, 451 (7175), 168 (2008).

[11] Е.И. Гиваргизов. Природа, 11, 20 (2003).

[12] H. Jagannathan, Y. Nishi. Appl. Phys. Lett., 88, 103113 (2006).

[13] В.А. Небольсин, Д.Б. Суятин, Е.В. Зотова, С.С. Шмакова. Неорг. матер., 48 (9), 979 (2012). 
[14] A. Kaushik, R. Kumar, E. Huey, S. Bhansali, E. Nair, M. Nair. Microchim. Acta, 181, 1759 (2014).

[15] Q. Wei, G. Meng, X. An, Y. Hao, L. Zhang. Sol. St. Commun., 138, 325 (2006).

[16] V.A. Nebol'sin, J. Johansson, D.B. Suyatin, B.A. Spiridonov. Cryst. Growth, 505, 52 (2019).

[17] D.P. Yu, Q.L. Hang, Y. Ding, H.Z. Zhang, Z.G. Bai, J.J. Wang, Y.H. Zou, W. Qian, G.C. Xiong, S.Q. Feng. Appl. Phys. Lett., 73, 3076 (1998).

[18] L. Dai, L.P. You, X.F. Duan, W.C. Lian, G.G. Qin. Phys. Lett. A, 335, 304 (2005).

[19] В.А. Небольсин, А.Ю. Воробьев, N. Swaikat. Неорг. матер., 56 (4), 364 (2020).

[20] P.K. Sekhar, S.N. Sambandam., D.K. Sood, S. Bhansali. Nanotechnology, 17, 4606 (2006).

Редактор Г.А. Оганесян

\section{Influence of the Oxide Surface Layer \\ on the Growth of Epitaxial Silicon Nanowires}

V.A. Nebolsin, N.A. Swaikat, A.Yu. Vorobiev,

T.A. Perepechina, L.V. Ozhogina

Voronezh State Technical University, 394026 Voronezh, Russia

Abstract It was found that when growing $\mathrm{Si}$ nanowires in an $\mathrm{H}_{2}$ flow, which was not subjected to additional purification from residues of $\mathrm{O}_{2}$ and water vapor, $\mathrm{SiO}_{2}$ layers are formed on the crystal surface and on the growth substrate. Due to the presence of an oxide surface layer, the growth of Si nanowires is inhibited, and the crystals are characterized by strong morphological instability. The thermodynamic conditions for the formation of a surface oxide layer and its effect on the growth of $\mathrm{Si}$ are determined. At synthesis temperatures of $750-1400 \mathrm{~K}, \mathrm{Si}$ nanowires are thermodynamically unstable in the gas phase containing any appreciably low $\mathrm{O}_{2}$ concentrations, and under favorable kinetic conditions, $\mathrm{Si}$ should completely transform into oxide. Thermal dissociation and hydrogen reduction of $\mathrm{SiO}_{2}$ under the conditions of $\mathrm{Si}$ nanowires growth are practically unrealizable. 